\title{
Delayed tension pneumothorax complicating staphylococcal pneumonia
}

\author{
S.P. Yates and S.K. Morcos \\ Department of Radiodiagnosis and Imaging, Northern General Hospital, Herries Road, Sheffield S5 $7 A U$, \\ $U K$.
}

\begin{abstract}
Summary: A case is described in which a tension pneumothorax complicated staphylococcal pneumonia 11 months after its onset. The delayed and subacute/chronic nature of the tension pneumothorax is unusual. The case also highlights the difficult differential diagnosis between subpleural lung cysts and encysted pneumothorax.
\end{abstract}

\section{Introduction}

Staphylococcal pneumonia is fairly uncommon outside hospitals, where it accounts for between 1 and $15 \%$ of acute pneumonias. ${ }^{1,2}$ It is rare for staphylococci to cause pneumonia in previously healthy individuals. Those who have viral lower respiratory tract infections are predisposed, as are the immunocompromised. ${ }^{2}$ Pulmonary infection may follow septicaemia due to distant focus of staphylococcal infection, but when this is the case the pattern tends to be one of discrete but multiple lung abscesses. ${ }^{3}$ The more usual pattern is that of a widespread bronchopneumonia, though occasionally the pneumonia can be confined to a single lobe or segment. ${ }^{4}$ Cavitation of the consolidated areas of the lung may occur and resolution of the pneumonia is generally complete, with little or no scarring. Rupture of staphylococcal abscess cavities has been recognized as a cause of pneumothorax during the acute illness. ${ }^{3}$ The spread of sepsis to the pleural cavity can result in empyema and permanent damage to the pleura, with thickening and adhesions.

We report a patient with a Staphylococcus aureus pneumonia who slowly developed a tension pneumothorax over a period of 11 months. The early radiological appearances were wrongly interpreted as pneumatocoeles.

\section{Case report}

A 66 year old woman suffering from congestive cardiomyopathy was admitted with a chest infection and extensive consolidation of the right lung

Correspondence: S.K. Morcos F.R.C.S., F.R.C.R.

Accepted: 4 May 1988.

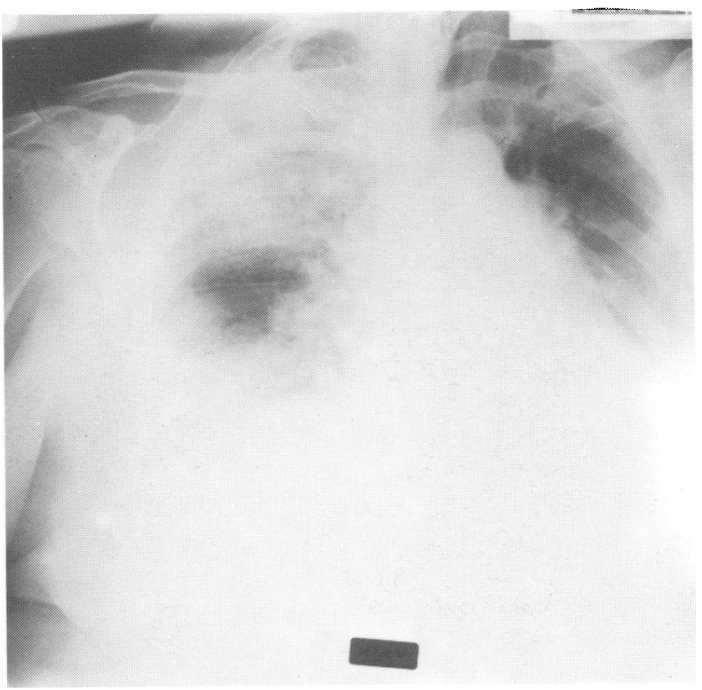

Figure 1 A chest radiograph demonstrates extensive consolidation in the right lung.

(Figure 1). Staphylococcus aureus was isolated from the sputum. As the initial response to antibiotics was poor, fibreoptic bronchoscopy was performed. This revealed thick purulent mucus in the right bronchial tree but no evidence of an obstructing lesion. Cavitation developed in some of the consolidated areas of the right lung (Figure 2). The pneumonia was slow to resolve but 3 months after the onset of the illness there was full clinical recovery. Several cavities remained in the right lung, and these were erroneously interpreted as pneumatocoeles (Figure 3).

The patient defaulted from follow-up and 8 months later she was referred with dyspnoea which

(C) The Fellowship of Postgraduate Medicine, 1988 


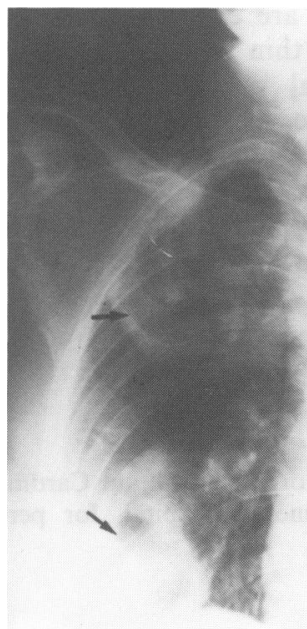

Figure 2 Cavitation (arrows) developed within the consolidated areas of the right lung.

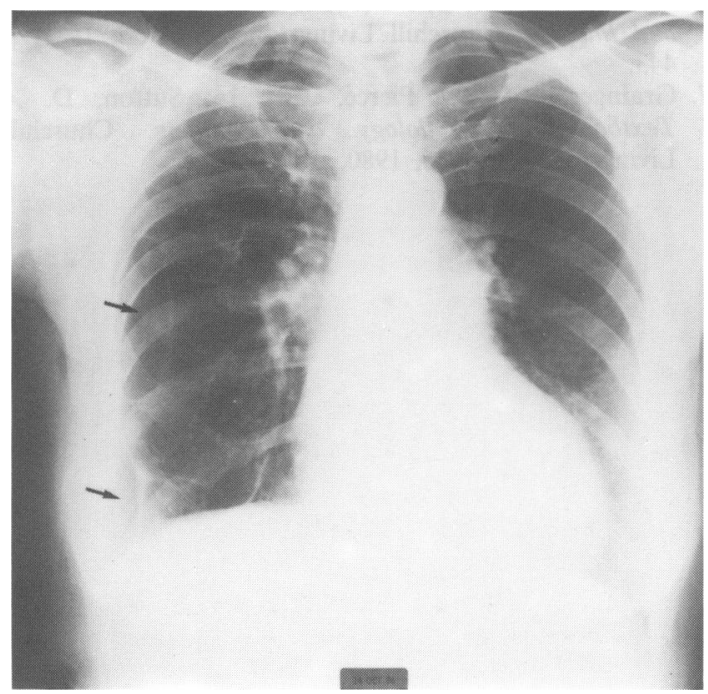

Figure 3 Thin walled cysts (arrows) seen in the right mid and lower zones.

was slowly progressive but disabling. There was no complaint of chest pain. The chest radiograph showed complete replacement of the right lung by multiple thin walled cavities, and considerable mediastinal shift to the left (Figure 4). Thoracotomy was carried out to relieve the tension. At operation a large multi-loculated pneumothorax was found, with complete collapse of the right lung. The right lung was re-expanded and pleurectomy performed to prevent recurrence. Slow but complete

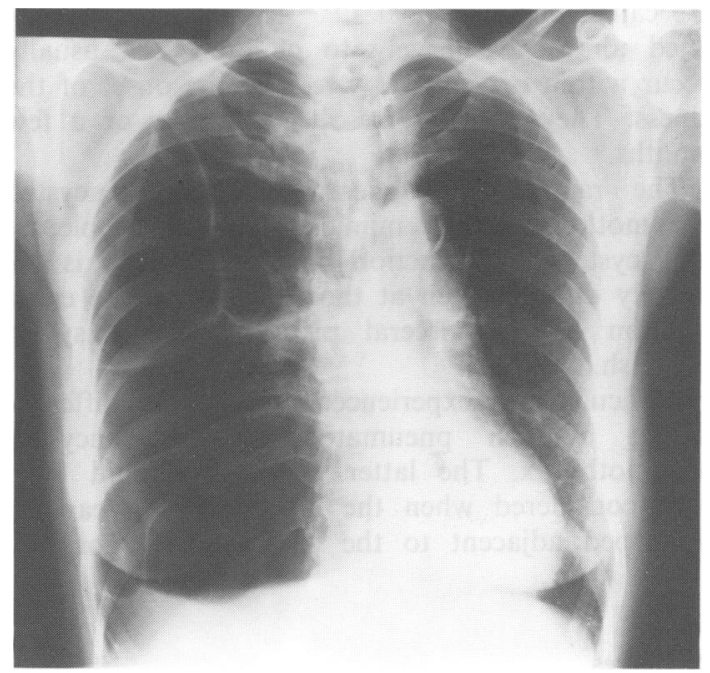

Figure 4 Multiple large thin walled cavities with tension in the right hemithorax, causing displacement of the heart and mediastinum to the left.

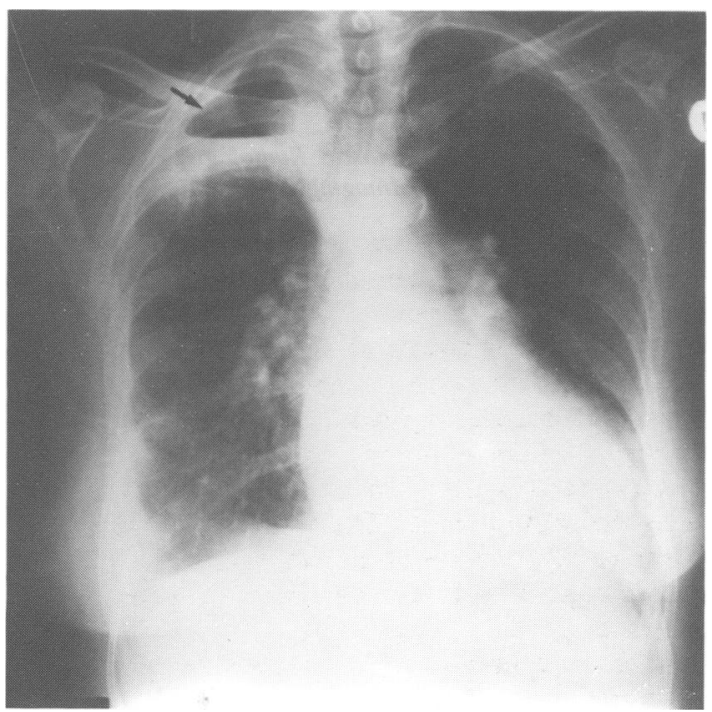

Figure 5 Eight weeks after surgery a small right apical hydropneumothorax persists (arrow).

recovery followed the operation, and a radiograph taken 8 weeks post-operatively shows almost complete re-expansion of the right lung, with a persistent small apical hydropneumothorax (Figure 5).

\section{Discussion}

Patients with staphylococcal pneumonia may develop thin walled cavities within the lungs which 
are called pneumatocoeles. ${ }^{1,5}$ These cysts are confined almost exclusively to children and usually occur within one or two weeks of the onset of the illness. They normally resolve in weeks or a few months. ${ }^{6}$

The radiological appearance of an encysted pneumothorax closely mimicks that of a subpleural lung cyst. The distinction between the two is extremely difficult even at thoracotomy as the exact position of the visceral pleura is not easy to establish. ${ }^{7}$

Difficulty was experienced in our case differentiating between pneumatocoeles and encysted pneumothorax. The latter diagnosis should have been considered when the air containing cavities developed adjacent to the chest wall (Figure 3),

\section{References}

1. Genereux, G.P. \& Stillwell, G.A. The acute bacterial pneumonias. Seminars in Roentgenology 1980, 15: 9-16.

2. Naraqi, S. \& McDonnell, G. Haematogenous staphylococcal pneumonia secondary to soft tissue infection. Chest 1981, 79: 173-175.

3. Jaffe, R.B. \& Koschmann, E.B. Septic pulmonary emboli. Radiology 1970, 96: 527-532.

4. Alford, R.A. \& Keats, T.E. In: Grainger, R.G. \& Allison, D.J. (eds) Diagnostic Radiologv - an AngloAmerican Textbook of Imaging. Churchill Livingstone, London, 1986, pp 337. especially as pneumatocoeles are extremely rare in adults. Patients who develop thin walled cavities in the course of staphylococcal pneumonia require careful follow-up until there has been complete resolution of the radiological changes. Careful monitoring of these patients is essential for the early detection of tension pneumothorax, which could be fatal if unrecongized.

\section{Acknowledgement}

We wish to thank Mr J.A.C. Thorpe, Consultant Cardiothoracic Surgeon, Northern General Hospital, for permission to report this case.

5. Highman, J.H. Staphylococcal pneumonia and empyema in childhood. Am J Roentgenol 1969, 106: 103-108.

6. Hyde, I. In: Sutton, D. (ed) A Textbook of Radiology and Imaging. Churchill Livingstone, London, 1987, $\mathrm{p}$ 443.

7. Grainger, R.G. \& Pierce, J.W. In: Sutton, D. $A$ Textbook of Radiology and Imaging. Churchill Livingstone, London, 1980, p 285. 